\title{
Translating pharmacogenomics discoveries into the clinic: an implementation framework
}

\author{
R Stephanie Huang ${ }^{1 *}$ and Eric R Gamazon ${ }^{2}$ \\ See related Research paper, http://genomemedicine.com/content/5/10/93
}

\begin{abstract}
The implementation of pharmacogenomics-guided care has the potential to improve clinical outcomes; however, the adoption of pharmacogenomics into clinical practice remains slow. This is partly due to the lack of a standardized framework for translating key findings into diagnostic tests. A recent study describes a comprehensive system developed by the Coriell Personalized Medicine Collaborative for facilitating the translation of genomic findings into clinical practice, and its application for seven commonly prescribed drugs.
\end{abstract}

In recent years, as advances in technologies have facilitated the generation of genomic information, we have witnessed an unprecedented explosion of pharmacogenomic discoveries. However, only a small number of these discoveries have made their way into clinical practice. There are several barriers to successful clinical implementation. First, a high-quality genotyping assay with rapid turnaround time is required. Second, the appropriate interpretation of pharmacogenetic test results by busy clinicians requires an adequate clinical decision support infrastructure, and the rapid advances in the field of pharmacogenomics may underscore the need for adequate training for healthcare professionals [1]. Last, the absence of a standardized framework for incorporating pharmacogenomic discoveries into diagnostic tests and therapeutic strategies is currently a major obstacle. Among the efforts aimed at addressing this gap, a recent article by Gharani and colleagues [2], published in Genome Medicine, presents the Pharmacogenomics Appraisal, Evidence Scoring and Interpretation System (PhAESIS), and its application to seven commonly prescribed drugs.

\footnotetext{
* Correspondence: rhuang@medicine.bsd.uchicago.edu

${ }^{1}$ Section of Hematology/Oncology, Department of Medicine, University of Chicago, Chicago, IL 60637, USA

Full list of author information is available at the end of the article
}

\section{The PhAESIS framework}

PhAESIS is a multi-purpose framework that identifies genetic factors with sufficiently robust evidence for contributing to drug response and provides guidelines for translating such genotype information into therapeutic recommendations. PhAESIS was developed by the Coriell Personalized Medicine Collaborative (CPMC) to guide the identification of genetic variants that are significantly associated with an elevated risk of adverse drug reactions; the ultimate goal is to translate pharmacogenomic findings into tailored therapy for patients. Genetic factors are identified based on a critical appraisal of pharmacologic studies. Furthermore, the framework provides recommendations for the clinical implementation of study findings and identifies gaps in knowledge for future investigation.

As the authors acknowledge, CPMC is not alone in its efforts to develop a system that converts the enormous number of published pharmacogenomic studies into clear and evidence-based therapeutic guidelines for clinical practice. Several well-known consortia, including the Clinical Pharmacogenetics Implementation Consortium (CPIC) [3], the Pharmacogenetics Working Group (PWG) [4], and Evaluation of Genomic Applications in Practice and Prevention (EGAPP) [5], are also developing guidelines. Each consortium has its strengths and weaknesses, some of which are covered in the study by Gharani et al. The CPMC framework aims to reflect a broader representation of the parties involved (for example, healthcare professionals and health consumers) and a more refined definition of the clinical relevance of pharmacogenomic findings (such as the distinction between genetic variants with published clinical outcome data and genetic variants with in vitro support for protein function).

There are opportunities for improvement. For example, the approach, in its current form, is quite gene- and function-centric. Only genetic variants deemed functional or located in genes were selected in the scoring system. It is of course true that most pharmacogenomic studies that have shown clinical relevance have, to date, focused 
primarily on genes. However, this merely reflects the history of pharmacogenomic research and may not be biologically informed. Indeed, large numbers of non-coding genetic variants have now been shown to be reproducibly associated with clinical phenotypes [6] by genome-wide association studies, although their underlying mechanisms remain to be fully elucidated. Therefore, the inclusion of such genetic variants into the PhAESIS system is highly desirable from a clinical standpoint and likely to be necessary even as we await their full characterization. Furthermore, we believe PhAESIS must eventually come to terms with the growing importance of high-throughput molecular phenotypes (for example, quantitative measurements of the transcriptome or the proteome) and their potential implications for diagnostic and clinical use.

\section{Future implications for clinical practice}

In this age of rapid advances in genomic research, it is critical to develop a precise and standardized approach to identify clinically relevant genomic loci and facilitate the translation of this information into the clinic. The study by Gharani et al. is therefore very timely. There are several clear strengths in the work presented. For example, the inclusion of a multi-tier evidence scoring system that enables a comprehensive appraisal of the clinical evidence and the routine incorporation of pharmacogenomic findings into clinical practice are primary emphases of the framework. Detailed information provided in the supplemental materials may be useful in providing guidelines for specific drug-gene cases. The authors have demonstrated the application of PhAESIS for the identification of key genes associated with adverse reactions for seven widely prescribed drugs (clopidogrel and CYP2C19; warfarin and CYP2C9, VKORC1 and CYP4F2; proton pump inhibitors and CYP2C19; codeine and CYP2D6; thiopurines and TPMT; simvastatin and SLCO1B1; and celecoxib and CYP2C9). The example the authors provide of a deferral on a wellknown drug-gene pair demonstrates the high quality of the evaluation process for selecting genetic variants, and the ability of the framework to incorporate increasing amounts of new evidence from studies. Furthermore, the sustained attention devoted to the gaps in pharmacogenomic knowledge, such as the limited information we have on the association between rare variants and adverse drug reactions, and the recognition of the need to expand pharmacogenomic studies to pharmacogenetically under-studied populations [7] highlight the comprehensiveness of the framework.

In summary, the work presented by Gharani et al. makes a welcome addition to the literature on harnessing the enormous advances in genomics in the clinical setting.
Appraisal, Evidence Scoring and Interpretation System;

PWG: Pharmacogenetics Working group.

\section{Competing interests}

The authors declare that they have no competing interests.

\section{Acknowledgements}

RSH received support from NIH/NIGMS grant K08GM089941, a Circle of Service Foundation Early Career Investigator award, University of Chicago Cancer Center Support Grant (\#P30 CA14599), Breast Cancer SPORE Career Development Award (CA125183), NIH/NCI grant R21 CA139278, NIH/NIGMS grant U01GM61393, a Conquer Cancer Foundation of ASCO Translational Research Professorship award in memory of Merrill J Egorin, MD, and a pilot grant from NIH/NCATS grant UL1RR024999.

\section{Author details}

${ }^{1}$ Section of Hematology/Oncology, Department of Medicine, University of Chicago, Chicago, IL 60637, USA. ${ }^{2}$ Section of Genetic Medicine, Department of Medicine, University of Chicago, Chicago, IL 60637, USA.

\section{Published: 18 October 2013}

\section{References}

1. Huang RS, Ratain MJ: Pharmacogenetics and pharmacogenomics of anticancer agents. CA Cancer J Clin 2009, 59:42-55.

2. Gharani N, Keller MA, C.B. S, Hodges LM, Schmidlen TJ, et al: The coriell personalized medicine collaborative pharmacogenomics appraisal, evidence scoring and interpretation system. Genome Med 2013, 5:93.

3. Relling MV, Klein TE: CPIC: Clinical Pharmacogenetics Implementation Consortium of the Pharmacogenomics Research Network. Clin Pharmacol Ther 2011, 89:464-467.

4. Swen JJ, Nijenhuis M, de Boer A, Grandia L, Maitland-van der Zee AH, Mulder H, Rongen GA, van Schaik RH, Schalekamp T, Touw DJ, van der Weide J, Wilffert B, Deneer VH, Guchelaar HJ: Pharmacogenetics: from bench to byte - an update of guidelines. Clin Pharmacol Ther 2011, 89:662-673.

5. Teutsch SM, Bradley LA, Palomaki GE, Haddow JE, Piper M, Calonge N, Dotson WD, Douglas MP, Berg AO, EGAPP Working Group: The Evaluation of Genomic Applications in Practice and Prevention (EGAPP) initiative: methods of the EGAPP Working Group. Genet Med 2009, 11:3-14.

6. Hindorff LA, Sethupathy P, Junkins HA, Ramos EM, Mehta JP, Collins FS, Manolio TA: Potential etiologic and functional implications of genomewide association loci for human diseases and traits. Proc Natl Acad Sci USA 2009, 106:9362-9367.

7. Gamazon ER, Perera M: Genome-wide approaches in pharmacogenomics: heritability estimation and pharmacoethnicity as primary challenges. Pharmacogenomics 2012, 13:1101-1104.

doi:10.1186/gm497

Cite this article as: Huang and Gamazon: Translating pharmacogenomics discoveries into the clinic: an implementation framework. Genome Medicine 2013 5:94

\section{Abbreviations}

CPIC: Clinical Pharmacogenetics Implementation Consortium; CPMC: Coriell

Personalized Medicine Collaborative; EGAPP: Evaluation of Genomic

Applications in Practice and Prevention; PhAESIS: Pharmacogenomics 